\title{
Applications of the Trace Formula
}

\author{
A. W. Knapp and J. D. Rogawski
}

This paper is an introduction to some ways that the trace formula can be applied to proving global functoriality. We rely heavily on the ideas and techniques in [Kn1], [Kn2], and [Ro4] in this volume.

To address functoriality with the help of the trace formula, one compares the trace formulas for two different groups. In particular the trace formula for a single group will not be enough, and immediately the analysis has to be done in an adelic setting. The idea is to show that the trace formulas for two different groups are equal when applied respectively to suitably matched functions. The actual matching is a problem in local harmonic analysis, often quite difficult and as yet not solved in general. The equality of the trace formulas and some global analysis allow one to prove cases of functoriality of automorphic representations. We discuss three examples in the first three sections - the Jacquet-Langlands correspondence, existence of automorphic induction in a special case, and aspects of base change.

A feature of this kind of application is that it is often some variant of the trace formula that has to be used. In our applications we use the actual trace formula in the first example, a trace formula that incorporates an additional operator in the second example, and a "twisted trace formula" in the third example. The paper [Ja] in this volume describes a "relative trace formula" in this context, and [Ar2] describes the need for a "stable trace formula" and a consideration of "endoscopy." See [Ar-Cl], [Bl-Ro], [Lgl-Ra], [Ra], and [Ro2] for some advanced applications.

For completeness we mention that the trace formula has another kind of application that is not closely related to functoriality. In this case one makes an absolute use of the trace formula for a single group. We discuss briefly three examples in the last section - the Eichler-Selberg trace formula, a property of the multiplicative groups of quaternion algebras, and a consequence of the Eichler-Shimura congruence relation. The first and third of these involve $G L_{2}$ and are often discussed in the classical setting without adeles. However, we shall use adeles throughout.

The sections of the paper are as follows:

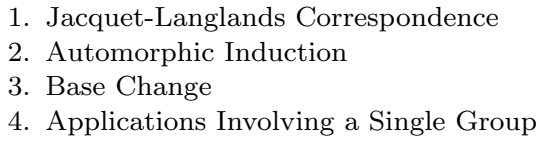

1991 Mathematics Subject Classification. Primary 11R39, 11R42, 22E45, 22E55. 
We use the following notation. The base field will be a number field $F, \bar{F}$ will be a fixed algebraic closure of $F$, and $\mathbb{A}$ will denote the adeles of $F$. If an extension field of $F$ appears, we shall use subscripts on $\mathbb{A}$ to distinguish the adeles of the various fields. In each application, $G$ will denote some particular reductive linear group over $F$, and $Z$ will denote the center. We fix a character $\omega$ of $Z(\mathbb{Q}) \backslash Z(\mathbb{A})$ and denote by $L^{2}(G, \omega)$ the space of functions $f$ on $G(\mathbb{A})$ that are left invariant under $G(\mathbb{Q})$, transform by $f(z g)=\omega(z) f(g)$ under $Z(\mathbb{A})$, and have $|f|$ square integrable on $Z(\mathbb{A}) G(\mathbb{Q}) \backslash G(\mathbb{A})$. Let $R$ be the right regular representation of $G(\mathbb{A})$ on $L^{2}(G, \omega)$. If $v$ is a place of $F$, we write $G_{v}$ and $Z_{v}$ for $G\left(F_{v}\right)$ and $Z\left(F_{v}\right)$.

We write $\bar{G}=Z \backslash G$. Let $C_{\mathrm{com}}^{\infty}\left(G(\mathbb{A}), \omega^{-1}\right)$ be the space of functions $\varphi$ on $G(\mathbb{A})$ that transform by $\varphi(z g)=\omega(z)^{-1} \varphi(g)$ for $z \in Z(\mathbb{A})$ and are smooth and have compact support modulo $Z(\mathbb{A})$. For $\varphi \in C_{\mathrm{com}}^{\infty}\left(G(\mathbb{A}), \omega^{-1}\right)$ and $f \in L^{2}(G, \omega)$, we set

$$
R(\varphi) f(x)=\int_{\bar{G}(\mathbb{A})} f(x y) \varphi(y) d y
$$

the integrand is well defined as a function of $y \in \bar{G}(\mathbb{A})$.

\section{Jacquet-Langlands Correspondence}

For the Jacquet-Langlands correspondence let $G=G L_{2}$ over $F$, and let $G^{\prime}$ be the multiplicative group of a quaternion algebra $D$ over $F$, i.e., a four-dimensional central division algebra over $F$. Quaternion algebras and the algebraic group $G^{\prime}$ are discussed in this volume in $[\mathrm{Kn} 2, \S 5]$. See also [Ro3] and [Vi].

In particular the quotient $Z^{\prime}(\mathbb{A}) G^{\prime}(\mathbb{Q}) \backslash G^{\prime}(\mathbb{A})$ is compact. Let $S$ be the finite set of places $v$ where $G_{v}^{\prime}=G^{\prime}\left(F_{v}\right)$ is ramified, i.e., where $D \otimes_{F} F_{v}$ remains a division algebra. It is known that $S$ is not empty and contains an even number of places. At the remaining places we say that $G_{v}^{\prime}$ is split.

The $L$ groups of $G$ and $G^{\prime}$ in the sense of $[K n 1, \S 9]$ are both $G L_{2}(\mathbb{C}) \times \Gamma$, where $\Gamma=\operatorname{Gal}(\bar{F} / F)$. The case of global functoriality that is addressed by the Jacquet-Langlands correspondence is the identity map ${ }^{L} G^{\prime} \rightarrow{ }^{L} G$.

Let us consider this map at the local level, where an example will be helpful. When $F=\mathbb{Q}, G_{\infty}^{\prime}$ is either $G L_{2}(\mathbb{R})$ or the multiplicative group $\mathbb{H}^{\times}$of the ordinary quaternions, and in either case the $L$ group is the direct product of $G L_{2}(\mathbb{C})$ and the two-element Galois group $\operatorname{Gal}(\mathbb{C} / \mathbb{R})$. However, the admissible homomorphisms of the Weil group $W_{\mathbb{R}}$ into the two $L$ groups are different. In the first case the definition is as in $[\mathrm{Kn} 2, \S 9]$, but in the second case, $[\mathrm{Kn} 2, \S 9]$ noted that another condition needs to be satisfied. That condition involves "relevance" of parabolic subgroups of ${ }^{L} G^{\prime}$ and is discussed in [Bo, pp. 32, 40]. In this way the local Langlands correspondence for the quaternion case leads only to the familiar representations of the compact-mod-center group $G_{\infty}^{\prime}=S U(2) \times \mathbb{R}_{+}^{\times}$, while the local Langlands correspondence for $G_{\infty}=G L_{2}(\mathbb{R})$ leads both to discrete series and to principal series. At the local level, functoriality in the quaternion case matches the familiar representations of $S U(2) \times \mathbb{R}_{+}^{\times}$with the discrete series of $G L_{2}(\mathbb{R})$. See Remark 1 after Theorem 1a below for more detail.

Imagine piecing together local identifications of this kind for each place and obtaining an identification of irreducible admissible representations of $G^{\prime}(\mathbb{A})$ with

some of those of $G(\mathbb{A})$. The assertion of global functoriality is that automorphic representations correspond to automorphic representations. 
Let us state precise results as theorems. We begin with the local case. If $k$ is a local field of characteristic 0 and $A$ is a quaternion division algebra over $k$, then there exist a number field $F$ and a division algebra $D$ over $F$ such that $F_{v}=k$ and $D(k)=A$. Because of this circumstance, there is no loss of generality in the local case in stating our results for $F_{v}$ and a ramified $G_{v}^{\prime}$.

In this situation consider regular semisimple elements $t_{v}^{\prime}$ and $t_{v}$ in $G_{v}^{\prime}$ and $G_{v}$. We write $t_{v}^{\prime} \sim t_{v}$ if $t_{v}^{\prime}$ and $t_{v}$ "have the same eigenvalues," i.e., if the traces and determinants of $t_{v}^{\prime}$ as a 2-by-2 matrix as in $[\mathrm{Kn} 2, \S 5]$ match those for $t_{v}$. The relation $t_{v}^{\prime} \sim t_{v}$ defines a bijection between noncentral conjugacy classes in $G_{v}^{\prime}$ and the noncentral semisimple conjugacy classes in $G_{v}$ that are not hyperbolic, i.e., do not have eigenvalues in $F_{v}$.

We can now define the local Jacquet-Langlands correspondence. With $G_{v}^{\prime}$ ramified, let $\pi_{v}^{\prime}$ and $\pi_{v}$ be irreducible admissible representations of $G_{v}^{\prime}$ and $G_{v}$, respectively, and let $\theta_{\pi_{v}^{\prime}}$ and $\theta_{\pi_{v}}$ be their global characters. We say that $\pi_{v}^{\prime}$ and $\pi_{v}$ correspond, written $\pi_{v}^{\prime} \leftrightarrow \pi_{v}$, if $\theta_{\pi_{v}^{\prime}}\left(t^{\prime}\right)=-\theta_{\pi_{v}}(t)$ for all regular semisimple elements $t^{\prime} \in G_{v}^{\prime}$ and $t \in G_{v}$ such that $t_{v}^{\prime} \sim t_{v}$.

Preliminary REMARKS.

(1) Let us say that an irreducible admissible representation $\pi_{v}$ of $G_{v}$ is elliptic if its character $\theta_{\pi_{v}}$ does not vanish identically on the elliptic set (an elliptic element for this $G$ being one with eigenvalues not in $F$ ). It is clear that $\pi_{v}$ can correspond to some $\pi_{v}^{\prime}$ only if $\pi_{v}$ is elliptic.

(2) If $\pi_{v}^{\prime} \leftrightarrow \pi_{v}$, then the central characters of $\pi_{v}^{\prime}$ and $\pi_{v}$ match on the centers $F_{v}^{\times}$of $G_{v}^{\prime}$ and $G_{v}$.

(3) Since $\pi_{v}^{\prime}$ is finite-dimensional, it is determined by its global character $\theta_{\pi_{v}^{\prime}}$. It follows that a given $\pi_{v}$ corresponds to at most one $\pi_{v}^{\prime}$. It is also true, but less obvious, that $\pi_{v}^{\prime}$ corresponds to at most one $\pi_{v}$. This follows from the fact that an elliptic representation of $G L_{2}\left(F_{v}\right)$ is determined by the restriction of its character to the elliptic set, which in turn follows from orthogonality relations for the global characters on the elliptic set ([Ja-Lgl]).

Theorem 1a. The local Jacquet-Langlands correspondence is a bijection between the set $\Pi\left(G_{v}^{\prime}\right)$ of classes of irreducible admissible representations of $G_{v}^{\prime}$ and the set $\Pi^{2}\left(G_{v}\right)$ of classes of discrete series representations of $G_{v}$.

REMARKS.

(1) If $F_{v}=\mathbb{R}$ and $G_{v}^{\prime}$ is ramified, then $G_{v}^{\prime}=S U(2) \times \mathbb{R}_{+}^{\times}$, while $G_{v}=G L_{2}(\mathbb{R})$ is the product of the group $S L_{2}^{ \pm}(\mathbb{R})$ of determinant \pm 1 by $\mathbb{R}_{+}^{\times}$. By the unitary trick each irreducible finite-dimensional representation $\pi_{v}^{\prime}$ of $S U(2)$ complexifies to a holomorphic representation of $S L_{2}(\mathbb{C})$ and then restricts to an irreducible finitedimensional representation of $S L_{2}(\mathbb{R})$. To such a finite-dimensional representation $V_{n}$ of $S L_{2}(\mathbb{R})$ of dimension $n$, there are two discrete series representations $\mathcal{D}_{n+1}^{+}$ and $\mathcal{D}_{n+1}^{-}$such that $V_{n}, \mathcal{D}_{n+1}^{+}$, and $\mathcal{D}_{n+1}^{-}$are the irreducible subquotients of a principal series representation of $S L_{2}(\mathbb{R})$. The global character of the principal series representation is 0 on the usual compact Cartan subgroup, and thus the character of $V_{n}$ is the negative of the sum of the characters of $\mathcal{D}_{n+1}^{+}$and $\mathcal{D}_{n+1}^{-}$on this compact Cartan subgroup. Induction of either of $\mathcal{D}_{n+1}^{+}$or $\mathcal{D}_{n+1}^{-}$to $S L_{2}^{ \pm}(\mathbb{R})$ gives a discrete series representation $\mathcal{D}_{n+1}$ whose restriction to $S L_{2}(\mathbb{R})$ is $\mathcal{D}_{n+1}^{+} \oplus \mathcal{D}_{n+1}^{-}$. Then $V_{n} \mapsto \mathcal{D}_{n+1}$ is the local Jacquet-Langlands correspondence in this case. 
(2) Even though the one-dimensional representations of $G_{v}^{\prime}$ and $G_{v}$ in the theorem can be naturally identified, they do not match under the local JacquetLanglands correspondence. In fact, the characters of naturally identified onedimensional representations of $G_{v}^{\prime}$ and $G_{v}$ agree on the elliptic set, instead of being negatives of each other. When $v$ is nonarchimedean and $\pi_{v}^{\prime}$ is one-dimensional for $G_{v}^{\prime}$ of the form $\chi \circ$ det, the corresponding representation $\pi_{v}$ of $G_{v}$ is the special representation associated to $\chi$.

(3) The local Jacquet-Langlands correspondence was obtained originally in [Ja-Lgl], using properties of the Weil representation, and an exposition appears in [Gb1]. We shall proceed quite differently, bypassing the Weil representation and deducing the local result Theorem 1a from an intermediate step in the proof of a global result, Theorem 1b below. In doing so, we follow [Gb-Ja] and [Ro3].

(4) Theorem 1a addresses $G L_{2}$. An analogous result for $G L_{3}$ was obtained by Flath in his thesis, and a generalization to $G L_{n}$ was obtained in [DKV] and [Ro1]. The Weil representation no longer plays a role.

Let us turn to the global case. If $v$ is a place where $G_{v}^{\prime}$ is split, we say that $\pi_{v}^{\prime}$ and $\pi_{v}$ correspond, written $\pi_{v}^{\prime} \leftrightarrow \pi_{v}$, if they are equivalent under any fixed isomorphism $G_{v}^{\prime} \cong G_{v}$. This property is independent of the chosen isomorphism since every automorphism of $G L_{2}\left(F_{v}\right)$ is inner.

We can identify $Z^{\prime}(F) \backslash Z^{\prime}(\mathbb{A})$ canonically with $F^{\times} \backslash \mathbb{A}^{\times}$, and we can also identify $Z(F) \backslash Z(\mathbb{A})$ canonically with $F^{\times} \backslash \mathbb{A}^{\times}$. If the central character $\omega^{\prime}$ for $G^{\prime}$ corresponds to the central character $\omega$ for $G$ under these identifications, let us say that $\omega^{\prime}$ and $\omega$ match.

Let $\mathcal{A}\left(G^{\prime}\right)$ be the set of classes of automorphic representations of $G^{\prime}(\mathbb{A})$ with a unitary central character, and let $\mathcal{A}_{*}\left(G^{\prime}\right)$ be the set of those that are not onedimensional. Also let $\mathcal{A}_{0}^{S}(G)$ be the set of cuspidal representations $\pi=\bigotimes \pi_{v}$ of $G(\mathbb{A})$ with a unitary central character and with $\pi_{v}$ in the discrete series for every $v \in S$. The global Jacquet-Langlands correspondence is the map $\pi^{\prime} \mapsto \pi$ in the following theorem.

Theorem 1b. Let $\pi^{\prime}=\bigotimes \pi_{v}^{\prime}$ be in $\mathcal{A}_{*}(G)$, and let $\pi=\bigotimes \pi_{v}$ be the irreducible admissible representation of $G(\mathbb{A})$ such that $\pi_{v}^{\prime} \leftrightarrow \pi_{v}$ for all $v$. Then $\pi$ is in $\mathcal{A}_{0}^{S}(G)$. Furthermore the map $\pi^{\prime} \mapsto \pi$ is a bijection between $\mathcal{A}_{*}\left(G^{\prime}\right)$ and $\mathcal{A}_{0}^{S}(G)$.

Corollary (of proof). Each $\pi^{\prime}$ in $\mathcal{A}_{*}\left(G^{\prime}\right)$ occurs with multiplicity one.

This theorem appeared originally in [Ja-Lgl], and that book acknowledges the influence of earlier work by Shimizu; Theorem $1 \mathrm{~b}$ has its origins also in the work of Eichler. The proof shows that the global Jacquet-Langlands correspondence preserves multiplicities, and we know that multiplicity one holds for $G$; thus multiplicity one holds for $G^{\prime}$, as asserted in the corollary. Note that if $\pi^{\prime}$ and $\pi$ correspond as in Theorem 1b, then the definitions force the central character $\omega^{\prime}$ of $\pi^{\prime}$ to match the central character $\omega$ of $\pi$.

The line of proof of Theorems $1 \mathrm{a}$ and $1 \mathrm{~b}$ is to establish a global identity of traces by means of the trace formulas for $G^{\prime}$ and $G$ and to derive both theorems from this identity. The global identity says that there is a relationship between $R^{\prime}$ on $L^{2}\left(G^{\prime}, \omega^{\prime}\right)$ and $R$ on the discrete part of $L^{2}(G, \omega)$, i.e., the sum of the cuspidal part and the residual part, provided $\omega^{\prime}$ and $\omega$ match. If $\varphi^{\prime} \in C_{\mathrm{com}}^{\infty}\left(G^{\prime}(\mathbb{A}), \omega^{-1}\right)$ and $\varphi \in C_{\mathrm{com}}^{\infty}\left(G(\mathbb{A}), \omega^{-1}\right.$ ) are paired suitably (written $\varphi^{\prime} \leftrightarrow \varphi$ ), the relationship is that

$$
\operatorname{Tr}\left(R^{\prime}\left(\varphi^{\prime}\right)\right)=\operatorname{Tr}\left(P_{\text {cusp }} R(\varphi) P_{\text {cusp }}\right)+\operatorname{Tr}\left(P_{\text {res }} R(\varphi) P_{\text {res }}\right),
$$


where $P_{\text {cusp }}$ and $P_{\text {res }}$ are orthogonal projections.

Let us define the notion of "matching orbital integrals." First we consider this notion locally. Recall that $\bar{G}^{\prime}=Z^{\prime} \backslash G^{\prime}$ and $\bar{G}=Z \backslash G$. Fix $v$, and let Haar measures on $\bar{G}_{v}^{\prime}$ and $\bar{G}_{v}$ be given. We say that $\varphi_{v}^{\prime}$ and $\varphi_{v}$ have matching orbital integrals if the relevant conditions from the following list are satisfied:

(1) When $v$ is not in $S, \varphi_{v}^{\prime}=\varphi_{v}$ after $G_{v}^{\prime}$ has been identified with $G_{v}$.

(2) When $v$ is in $S$ and elliptic elements $t^{\prime}$ and $t$ are given with $t^{\prime} \sim t$, we can identify the centralizers $G_{v}^{\prime}{ }^{t^{\prime}}$ and $G_{v}{ }^{t}$ of $t^{\prime}$ and $t$ and then we can compatibly normalize the Haar measures on the quotients $\bar{G}_{v}^{\prime} t^{\prime} \backslash \bar{G}_{v}^{\prime}$ and $\bar{G}_{v}{ }^{t} \backslash \bar{G}_{v}$. In this situation we require

$$
\int_{\bar{G}_{v}^{\prime} t^{\prime} \backslash \bar{G}_{v}^{\prime}} \varphi_{v}^{\prime}\left(x^{-1} t^{\prime} x\right) d x=\int_{\bar{G}_{v} \backslash \bar{G}_{v}} \varphi_{v}\left(x^{-1} t x\right) d x \quad\left(t^{\prime} \sim t\right) .
$$

(3) When $v$ is in $S$ and a hyperbolic regular element $t$ is given in $G_{v}$, then

$$
\int_{\bar{G}_{v} \backslash \bar{G}_{v}} \varphi_{v}\left(x^{-1} t x\right) d x=0 .
$$

The local theorem about the existence of matching orbital integrals is that if $v$ is in $S$ and $\varphi_{v}^{\prime}$ is given, then there exists $\varphi_{v}$ with matching orbital integrals in the sense of (2) and (3) above. Moreover if $\varphi_{v}$ is given satisfying (3), then there exists $\varphi_{v}^{\prime}$ such that (2) holds. A proof of this result for nonarchimedean $v$ may be found in Section 2 of [Ro1]. For archimedean $v$, see [She].

For the global case we first fix Haar measures. The book [Ja-Lgl] describes a canonical way of defining Haar measure on $G_{v}^{\prime}$ once Haar measure on $G_{v}$ is given, and we use this normalization for every $v$. Then we say that $\varphi^{\prime}=\prod_{v} \varphi_{v}^{\prime}$ and $\varphi=\prod_{v} \varphi_{v}$ have matching orbital integrals (and we write $\varphi^{\prime} \leftrightarrow \varphi$ ) if $\varphi_{v}^{\prime}$ and $\varphi_{v}$ have matching orbital integrals for every $v$.

Under the assumption that $\varphi^{\prime} \leftrightarrow \varphi$, let us see why (1.1) follows. We shall use the trace formulas for $G^{\prime}$ and $G$. Let $M$ be the diagonal subgroup of $G$. From (1.2b) we have

$$
\int_{M_{v} \backslash G_{v}} \varphi_{v}\left(x^{-1}\left(\begin{array}{cc}
\alpha & 0 \\
0 & \beta
\end{array}\right) x\right) d x=0 \quad \text { for } v \in S
$$

whenever $\alpha$ and $\beta$ are distinct members of $F_{v}$. Since $S$ contains at least two places, Corollary 7.15 of [Kn2] says that several of the terms in the trace formula for $G$ vanish. More precisely, under the condition (1.3), Theorem 5.2 and Corollary 7.15 of [Kn2] give

$$
\operatorname{Tr}\left(R^{\prime}\left(\varphi^{\prime}\right)\right)=\sum_{\mathfrak{o}_{\gamma^{\prime}}} \operatorname{vol}\left(\bar{G}^{\prime}(F)^{\gamma^{\prime}} \backslash \bar{G}^{\prime}(\mathbb{A})^{\gamma^{\prime}}\right) \int_{\bar{G}^{\prime}(\mathbb{A})^{\gamma^{\prime} \backslash \bar{G}^{\prime}(\mathbb{A})}} \varphi^{\prime}\left(x^{-1} \gamma^{\prime} x\right) d x
$$

and

$$
\begin{aligned}
\operatorname{Tr}\left(P_{\text {cusp }} R(\varphi) P_{\text {cusp }}\right)+ & \operatorname{Tr}\left(P_{\text {res }} R(\varphi) P_{\text {res }}\right) \\
= & \operatorname{vol}(\bar{G}(F) \backslash \bar{G}(\mathbb{A})) \varphi(1) \\
& +\sum_{\substack{\mathfrak{o}_{\gamma} \\
\text { elliptic }}} \operatorname{vol}\left(\bar{G}(F)^{\gamma} \backslash \bar{G}(\mathbb{A})^{\gamma}\right) \int_{\bar{G}(\mathbb{A}) \gamma \backslash \overline{G(\mathbb{A})}} \varphi\left(x^{-1} \gamma x\right) d x,
\end{aligned}
$$


so that the two sides of (1.1) do resemble each other.

The right side of (1.4a) and the last term of (1.4b) are sums over $\left\{\gamma^{\prime}\right\}$ or $\{\gamma\}$ of products over $v$ of expressions

$$
m_{\gamma^{\prime}, v} \int_{\bar{G}_{v}^{\prime} \gamma^{\prime} \backslash \bar{G}_{v}^{\prime}} \varphi_{v}^{\prime}\left(x^{-1} \gamma^{\prime} x\right) d x \quad \text { and } \quad m_{\gamma, v} \int_{\bar{G}_{v} \gamma \backslash \bar{G}_{v}} \varphi_{v}\left(x^{-1} \gamma x\right) d x
$$

where $m_{\gamma^{\prime}, v}$ and $m_{\gamma, v}$ are certain local volume factors. These local volume factors have been arranged to match when $\gamma^{\prime} \sim \gamma$, and the orbital integrals match in this case by (1) and (2). Each $\gamma^{\prime}$ is $\sim \gamma$ for some $\gamma$. We have arranged by (3) that any $\gamma$ that is not $\sim \gamma^{\prime}$ for some $\gamma^{\prime}$ has orbital integral 0 ; indeed this follows from the fact that a quadratic extension $E$ embeds in the quaternion algebra if and only if no prime of $S$ splits in $E$.

In proving (1.1), we are left with the term in (1.4a) having $\gamma^{\prime}=1$ and the first term on the right side of $(1.4 \mathrm{~b})$. One shows that if global Haar measures are built from our matching local ones, then

$$
\operatorname{vol}\left(\bar{G}^{\prime}(F) \backslash \bar{G}^{\prime}(\mathbb{A})\right)=\operatorname{vol}(\bar{G}(F) \backslash \bar{G}(\mathbb{A})) .
$$

Moreover Harish-Chandra's formula for recovering the value of a function at 1 from its elliptic orbital integrals may be used to show that

$$
\varphi_{v}^{\prime}(1)=\left\{\begin{aligned}
-\varphi_{v}(1) & \text { for } v \in S \\
\varphi_{v}(1) & \text { for } v \notin S
\end{aligned}\right.
$$

Since $S$ contains an even number of places,

$$
\varphi^{\prime}(1)=\varphi(1)
$$

Thus the remaining terms on the right sides of (1.4a) and (1.4b) match, and (1.1) follows.

Letting $n\left(\pi^{\prime}\right)$ and $n(\pi)$ denote multiplicities, we obtain an equality

$$
\sum n\left(\pi^{\prime}\right) \operatorname{Tr}\left(\pi^{\prime}\left(\varphi^{\prime}\right)\right)=\sum n(\pi) \operatorname{Tr}(\pi(\varphi))
$$

when $\varphi^{\prime} \leftrightarrow \varphi$, where the sum on the left is over $\pi^{\prime}$ in $L^{2}\left(G^{\prime}, \omega^{\prime}\right)$ and the sum on the right is over cuspidal and residual $\pi$ in $L^{2}(G, \omega)$. The known multiplicity one for $G$ means that $n(\pi)$ is 1 when it is nonzero. Thus we can rewrite this equation as

$$
\sum n\left(\pi^{\prime}\right) \operatorname{Tr}\left(\pi^{\prime}\left(\varphi^{\prime}\right)\right)=\sum \operatorname{Tr}(\pi(\varphi))
$$

Digression. Before indicating how Theorems $1 \mathrm{a}$ and $1 \mathrm{~b}$ follow from (1.5), let us make an observation about a relationship between character identities and orbital integrals. From the Weyl integration formulas for integration over $G_{v}^{\prime}$ and $G_{v}$ and from the fact that irreducible characters are given by locally integrable functions, the trace of an irreducible admissible representation applied to a function is an integral of orbital integrals of the function. Thus if Theorem 1a is known (i.e., if $\pi_{v}$ has been constructed so as to be related to $\pi_{v}^{\prime}$ as in the theorem) and if $\varphi^{\prime} \leftrightarrow \varphi$, then it follows that

$$
\operatorname{Tr}\left(\pi_{v}^{\prime}\left(\varphi_{v}^{\prime}\right)\right)=\left\{\begin{aligned}
-\operatorname{Tr}\left(\pi_{v}\left(\varphi_{v}\right)\right) & \text { for } v \in S \\
\operatorname{Tr}\left(\pi_{v}\left(\varphi_{v}\right)\right) & \text { for } v \notin S
\end{aligned}\right.
$$


Since $S$ contains an even number of places, we obtain

$$
\operatorname{Tr}\left(\pi^{\prime}\left(\varphi^{\prime}\right)\right)=\operatorname{Tr}(\pi(\varphi)) .
$$

Then it is a fairly simple matter to use (1.5) to see that $\pi$ is automorphic. See [Gb-Ja, p. 248] for details. This is the approach of [Ja-Lgl], the local representations having been studied with the aid of the Weil representation.

Our task is different from the task in the above digression. We are to deduce both Theorem 1a and Theorem 1b from (1.5). The details are in [Gb-Ja, pp. 249-250] and in [Ro1], and our discussion will be brief.

In (1.5) let us repeat the term $\operatorname{Tr}\left(\pi^{\prime}\left(\varphi^{\prime}\right)\right)$ according to the multiplicity of $\pi^{\prime}$. Taking into account that $\varphi^{\prime}=\prod_{v} \varphi_{v}^{\prime}$ and $\varphi=\prod_{v} \varphi_{v}$, we can rewrite (1.5) as

$$
\sum \prod_{v} \operatorname{Tr}\left(\pi_{v}^{\prime}\left(\varphi_{v}^{\prime}\right)\right)=\sum \prod_{v} \operatorname{Tr}\left(\pi_{v}\left(\varphi_{v}\right)\right) .
$$

Lemma 1. For each $w \notin S$, fix an irreducible unitary representation $\tau_{w}$ of $G_{w}$. If $\varphi_{v}^{\prime}$ and $\varphi_{v}$ have matching orbital integrals for $v \in S$, then

$$
\sum \prod_{v \in S} \operatorname{Tr}\left(\pi_{v}^{\prime}\left(\varphi_{v}^{\prime}\right)\right)=\sum \prod_{v \in S} \operatorname{Tr}\left(\pi_{v}\left(\varphi_{v}\right)\right),
$$

where the sum on the left is over $\pi^{\prime}$ in $L^{2}\left(G^{\prime}, \omega^{\prime}\right)$ with $\pi_{w}^{\prime} \cong \tau_{w}$ for all $w \notin S$ and where the sum on the right is over cuspidal and residual $\pi$ in $L^{2}(G, \omega)$ with $\pi_{w} \cong \tau_{w}$ for all $w \notin S$. The sum on the right has at most one term.

The formula in Lemma 1 follows by applying to (1.6) a notion of generalized linear independence established in [Lab-L]. The sum on the right in (1.7) has at most one term as a consequence of strong multiplicity one for $G$.

Lemma 2 (Weak Jacquet-Langlands correspondence). If $\pi^{\prime}=\bigotimes \pi_{v}^{\prime}$ occurs in $L^{2}\left(G^{\prime}, \omega^{\prime}\right)$, then there exists a unique $\pi=\bigotimes \pi_{v}$ that is cuspidal or residual in $L^{2}(G, \omega)$ and has $\pi_{v}^{\prime} \cong \pi_{v}$ for all $v \notin S$. Moreover, $\pi_{v}$ is in the discrete series of $G_{v}$ for all $v \in S$. Conversely if $\pi=\bigotimes \pi_{v}$ is cuspidal in $L^{2}(G, \omega)$ is such that $\pi_{v}$ is in the discrete series of $G_{v}$ for all $v \in S$, then there exists at least one $\pi^{\prime}=\bigotimes \pi_{v}^{\prime}$ in $L^{2}\left(G^{\prime}, \omega^{\prime}\right)$ such that $\pi_{v}^{\prime} \cong \pi_{v}$ for all $v \notin S$.

SketCh OF PROOF. Uniqueness is by strong multiplicity one. For existence, suppose on the contrary that there is no $\pi$. Applying Lemma 1 with $\tau_{w}=\pi_{w}^{\prime}$ for all $w \notin S$, we see that

$$
\sum \prod_{v \in S} \operatorname{Tr}\left(\pi_{v}^{\prime}\left(\varphi_{v}^{\prime}\right)\right)=0
$$

where the sum is over $\sigma^{\prime}$ in $L^{2}\left(G^{\prime}, \omega^{\prime}\right)$ with $\sigma_{w}^{\prime} \cong \pi_{w}^{\prime}$ for all $w \notin S$. Generalized linear independence of characters [Lab-L] shows that the sum is empty, in contradiction to the fact that the sum must contain a term for each occurrence of $\pi^{\prime}$ in $L^{2}\left(G^{\prime}, \omega^{\prime}\right)$. The converse statement is proved in the same way, starting from the assumption that

$$
\sum \prod_{v \in S} \operatorname{Tr}\left(\pi_{v}\left(\varphi_{v}\right)\right)=0 .
$$

Lemma 2 gives the map $\pi^{\prime} \rightarrow \pi$ of Theorem 1b. Let us now turn our attention to Theorem 1a. Fix $\pi$ and take $\tau_{w}=\pi_{w}$ for $w \notin S$, so that the term for $\pi$ appears on the right side of (1.7). The first step is to show that the sum on the left side 
of (1.7) is a finite sum; the argument uses $\varepsilon$ factors and appears in Lemma 5.14 of [Ro1]. Then using linear independence of characters, we can omit from the product any place $v$ such that $\pi_{v}$ is a special representation (since we already know that this corresponds to a one-dimensional representation of $G_{v}^{\prime}$ ). We may also cancel the factors for archimedean $v \in S$, since we know the local Jacquet-Langlands correspondence for archimedean places. Thus we are left with a subset $S^{\prime} \subset S$ of places where each $\pi_{v}$ is supercuspidal. Then it follows that

$$
\sum \prod_{v \in S^{\prime}} \operatorname{Tr}\left(\pi_{v}^{\prime}\left(t^{\prime}\right)\right)=\prod_{v \in S^{\prime}} \operatorname{Tr}\left(\pi_{v}(t)\right) \quad \text { when } t^{\prime} \sim t .
$$

On the left side we have finitely many characters of the compact group $\prod_{v \in S} G_{v}^{\prime}$. The $L^{2}$ norm of the right side on the elliptic set is 1 by the orthogonality relations for supercuspidal characters on the elliptic set ([Ro1], Lemma 5.3). We conclude that there is only one summand $\pi^{\prime}$ on the left side, i.e.,

$$
\prod_{v \in S^{\prime}} \operatorname{Tr}\left(\pi_{v}^{\prime}\left(t^{\prime}\right)\right)=\prod_{v \in S^{\prime}} \operatorname{Tr}\left(\pi_{v}(t)\right) \quad \text { for some } \pi^{\prime} .
$$

Hence for each $v \in S^{\prime}$ there is a scalar $\lambda_{v}$ such that $\operatorname{Tr}\left(\pi_{v}^{\prime}\left(t^{\prime}\right)\right)=\lambda_{v} \operatorname{Tr}\left(\pi_{v}(t)\right)$. Again by the orthogonality relations, $\left|\lambda_{v}\right|=1$. To prove that $\lambda_{v}=-1$, we let $t^{\prime}$ tend to 1 . By continuity, $\operatorname{Tr}\left(\pi_{v}^{\prime}\left(t^{\prime}\right)\right)$ tends to $\operatorname{Tr}\left(\pi_{v}^{\prime}(1)\right)=\operatorname{dim}\left(\pi_{v}^{\prime}\right)$. By an argument in [Ro1], the supercuspidal $\pi_{v}$ has the properties that $\operatorname{Tr}\left(\pi_{v}(t)\right)$ is continuous at $t=1$ and $\operatorname{Tr}\left(\pi_{v}(1)\right)=-d\left(\pi_{v}\right)$, where $d\left(\pi_{v}\right)$ is the formal degree. Thus we obtain $\operatorname{dim}\left(\pi_{v}^{\prime}\right)=-\lambda_{v} d\left(\pi_{v}\right)$. Since $\operatorname{dim}\left(\pi_{v}^{\prime}\right)$ and $d\left(\pi_{v}\right)$ are positive, we conclude that $\lambda_{v}=-1$.

This completes the construction of the correspondence in Theorem 1a for all $\pi_{v}^{\prime}$ that occur as the $v^{\text {th }}$ component of some automorphic $\pi^{\prime}$ for $G^{\prime}$. But every $\pi_{v}^{\prime}$ arises in this way. In fact, Lemma 1.5 of [Ro1] shows that we have only to apply the trace formula to a function $\varphi^{\prime}=\prod \varphi_{w}^{\prime}$ of suitably small support with $\varphi_{v}^{\prime}$ equal to a matrix coefficient of $\pi_{v}^{\prime}$.

The rest of the argument for Theorems $1 \mathrm{a}$ and $1 \mathrm{~b}$ is relatively easy, and we omit the details.

\section{Automorphic Induction}

Automorphic induction is described in [Ro4, §15]. The point of departure is the question whether induction of Galois representations has a counterpart for automorphic representations. Let us suppose that $E / F$ is a finite extension of degree $d$ of number fields. For convenience we assume that $E / F$ is Galois. If $\sigma$ is an $m$-dimensional representation of $G_{E}=\operatorname{Gal}(\bar{F} / E)$, we form $\Sigma=\operatorname{ind}_{G_{E}}^{G_{F}} \sigma$ as a representation of $\operatorname{Gal}(\bar{F} / F)$ of degree $n=m d$. The $L$ functions of $\sigma$ and $\Sigma$ are equal. If the Local Langlands Conjecture holds at every place, then $\sigma$ parametrizes an irreducible admissible representation $\pi$ of $G L_{m}\left(\mathbb{A}_{E}\right)$ whose $L$ function equals the $L$ function of $\sigma$. Suppose $\pi$ is automorphic. Then $\Sigma$ parametrizes an irreducible admissible representation $\Pi$ of $G L_{n}\left(\mathbb{A}_{F}\right)$ with the same $L$ function, and the question is whether $\Pi$ is automorphic.

This situation is an instance of global functoriality. In fact, let

$$
H=R_{E / F}\left(G L_{m} / E\right) \quad \text { and } \quad G=G L_{n} / F
$$


where $R_{E / F}$ indicates restriction of scalars ( $\S \S 6$ and 10 of [Kn1]). Here $H(F)=$ $G L_{m}(E)$ and $H\left(\mathbb{A}_{F}\right)=G L_{m}\left(\mathbb{A}_{E}\right)$, and we have

$$
\begin{aligned}
{ }^{L} H & =\left(G L_{m}(\mathbb{C}) \times \cdots \times G L_{m}(\mathbb{C})\right) \rtimes G_{F} \quad\left(d \text { factors of } G L_{m}(\mathbb{C})\right), \\
{ }^{L} G & =G L_{n}(\mathbb{C}) \times G_{F},
\end{aligned}
$$

where $G_{F}$ acts on ${ }^{L} H$ by permuting the factors. The group $G_{F}$ acts through $\operatorname{Gal}(E / F)$, and $\operatorname{Gal}(E / F)$ may be regarded as a subgroup of the permutation group on $d$ letters. Regard the permutation for $\tau \in G_{F}$ as a $d$-by- $d$ matrix, and then replace each entry by an $m$-by- $m$ zero or identity matrix. In this way we can identify $\tau$ with an $n$-by-n matrix, which we denote $\tau$ also. The map ${ }^{L} H \rightarrow{ }^{L} G$ given by

$$
\left(\left(a_{1}, \ldots, a_{d}\right), \tau\right) \mapsto\left(\left(\begin{array}{ccc}
a_{1} & & \\
& \ddots & \\
& & a_{d}
\end{array}\right) \tau, \tau\right), \quad a_{j} \in G L_{m}(\mathbb{C}),
$$

is a homomorphism, and the global functoriality question is whether it corresponds to a map of automorphic representations.

As in $[$ Ro4, §15] we can rephrase the question in such a way that it is not necessary to assume the Local Langlands Conjecture. In fact, the given irreducible admissible representation $\pi$ of $H\left(\mathbb{A}_{F}\right)=G L_{m}\left(\mathbb{A}_{E}\right)$ has a tensor product decomposition $\pi=\bigotimes_{w} \pi_{w}$ in which $\pi_{w}$ is an unramified principal series for almost every place $w$ of $E$. For the unexceptional places $w$, the $L$ function $L\left(s, \pi_{w}\right)$ is well defined and determines $\pi_{w}$ up to isomorphism. Similar considerations apply to a representation $\Pi=\bigotimes_{v} \Pi_{v}$ of $G\left(\mathbb{A}_{F}\right)=G L_{n}\left(\mathbb{A}_{F}\right)$. Suppose $\pi$ is automorphic. We define $\Pi$ to be automorphically induced by $\pi$ if $\Pi$ is automorphic and

$$
L\left(s, \Pi_{v}\right)=\prod_{w \mid v} L\left(s, \pi_{w}\right)
$$

for almost all places $v$ of $F$. These conditions determine $\Pi$ uniquely by strong multiplicity one, since the local $L$ factors of $\Pi$ have already determined $\Pi_{v}$ at almost every place $v$. When $\Pi$ is automorphically induced by $\pi$, we write $\Pi=A I_{E}^{F}(\pi)$.

Theorem 2. Let $E / F$ be a cyclic extension of degree $d$. If $\pi$ is a cuspidal representation of $G L_{m}\left(\mathbb{A}_{E}\right)$, then $\Pi=A I_{E}^{F}(\pi)$ exists as an automorphic representation of $G L_{m d}\left(\mathbb{A}_{F}\right)$. Moreover, $\Pi$ is cuspidal unless there is a nontrivial element $\tau \in \operatorname{Gal}(E / F)$ such that $\tau(\pi)$ is isomorphic to $\pi$.

In this generality Theorem 2 is due to Arthur and Clozel [Ar-Cl]. We confine our comments to the special case that $d=2$ and $m=1$, which is the case that was handled originally. We shall write $\theta$ for the given automorphic representation $\pi$ of $G L_{1}\left(\mathbb{A}_{E}\right.$ ) (a unitary Grossencharacter of $E$ ) and $\Pi=\Pi_{\theta}$ for the automorphic representation of $G L_{2}\left(\mathbb{A}_{F}\right)$ that is to be constructed. We assume that $\theta \neq \theta^{\sigma}$, where $\sigma$ is the nontrivial element of $\operatorname{Gal}(E / F)$, and we are to construct $\Pi=\Pi_{\theta}$ and show that it is cuspidal automorphic.

Four methods are known for establishing this special case:

(1) Use theta series to construct $\Pi$ as a physical subspace of the appropriate $L^{2}$ space. This method is due to Hecke, Maass, and Shalika-Tanaka. 
(2) Use the Jacquet-Langlands converse theorem for $G L_{2}$. See Theorem 8.12 of [Kn1] for the statement. This is the method of Jacquet and Langlands [Ja-Lgl].

(3) Derive the result at the same time as establishing base change. This is the approach of Arthur and Clozel [Ar-Cl] and uses the trace formula.

(4) Use the trace formula in a different way. This is a method of Langlands [Lgl3].

We follow a variant of method (4). (See [Lab1].)

Thus let $E / F$ be a quadratic extension. By class field theory (particularly Theorem 4.7 in [Kn1]), there exists a nontrivial character $\alpha$ of $F^{\times} \backslash \mathbb{A}_{F}^{\times}$such that $\operatorname{ker} \alpha=N_{E / F}\left(E^{\times} \backslash \mathbb{A}_{E}^{\times}\right)$.

We are given a unitary Grossencharacter $\theta$ for $E$ (i.e., a character of $E^{\times} \backslash \mathbb{A}_{E}^{\times}$) that is not fixed by the nontrivial element $\sigma$ of $\operatorname{Gal}(E / F)$, and we are to construct an automorphic representation $A I_{E}^{F}(\theta)$ of $G L_{2}\left(\mathbb{A}_{F}\right)$.

We define an operator $A: L^{2}(G, \omega) \rightarrow L^{2}(G, \omega)$ by $A f(x)=f(x) \alpha(\operatorname{det} x)$. Note here that det $x$ is a square and hence lies in ker $\alpha=N_{E / F}\left(E^{\times} \backslash \mathbb{A}_{E}^{\times}\right)$if $x$ is in $Z\left(\mathbb{A}_{F}\right)$. We readily check that $A$ intertwines $R$ and $R \otimes(\alpha \circ$ det) in the sense that

$$
A(R \otimes(\alpha \circ \operatorname{det}))(x) f=R(x) A f \quad \text { for } f \in L^{2}(G, \omega) .
$$

Moreover, $A$ has order two and sends cuspidal functions to cuspidal functions. By strong multiplicity one for $G L_{2}$, the cuspidal irreducible representations in $L^{2}(G, \omega)$ have multiplicity one. If we think of the effect of $A$ on the irreducible summands $\Pi$ of the cuspidal part of $L^{2}(G, \omega)$, then we see that there are two possibilities:

(i) $\Pi \cong \Pi \otimes \alpha$, and then the space $V_{\Pi}$ of $\Pi$ is carried to itself by $A$

(ii) $\Pi \supsetneqq \Pi \otimes \alpha$, and then $A$ has in effect a matrix on the direct sum of the two

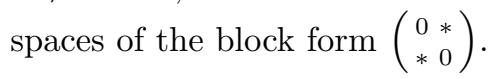

The same thing remains true of $P_{\text {cusp }} R(x) P_{\text {cusp }} A$ for every $x \in G\left(\mathbb{A}_{F}\right)$ and then also of $P_{\text {cusp }} R(\varphi) P_{\text {cusp }} A$ for every $\varphi \in C_{\text {com }}^{\infty}\left(G(\mathbb{A}), \omega^{-1}\right)$. The latter is of trace class since $P_{\text {cusp }} R(\varphi) P_{\text {cusp }}$ is of trace class, and we see that

$$
\operatorname{Tr}\left(P_{\text {cusp }} R(\varphi) P_{\text {cusp }} A\right)=\sum_{\substack{\Pi \cong \Pi \otimes \alpha, \Pi \text { cuspidal }}} \operatorname{Tr}\left(\Pi(\varphi) A_{\Pi}\right),
$$

where $A_{\Pi}$ is the restriction of $A$ to $V_{\Pi}$.

We shall want to use a variant of the trace formula to compute this trace in a different way. Part of the philosophy of applying the trace formula is that the main terms are the orbital integrals of the elliptic elements in $G(F)$ or $\bar{G}(F)$ (an elliptic element for this $G$ again being one with eigenvalues not in $F$ ). In order to isolate the elliptic terms, we write the unprojected operator as an integral operator and compute with the kernel as if the space of integration were compact. In the present case the operator $R(\varphi) A$ is an integral operator with kernel

$$
\sum_{\gamma \in \bar{G}(F)} \varphi\left(x^{-1} \gamma y\right) \alpha(\operatorname{det} y) .
$$


If the space of integration were compact, this operator would be of trace class, and a familiar computation shows that the trace of this operator would be

$$
\begin{aligned}
& =\sum_{\gamma \in \bar{G}(F)} \int_{\bar{G}(F) \backslash \bar{G}\left(\mathbb{A}_{F}\right)} \varphi\left(x^{-1} \gamma x\right) \alpha(\operatorname{det} x) d x \\
& =\sum_{\mathfrak{o}_{\gamma}} \int_{\bar{G}(F)^{\gamma} \backslash \bar{G}\left(\mathbb{A}_{F}\right)} \varphi\left(x^{-1} \gamma x\right) \alpha(\operatorname{det} x) d x,
\end{aligned}
$$

where $\mathfrak{o}_{\gamma}$ consists of all $\delta^{-1} \gamma \delta$ with $\delta$ varying through $\bar{G}(F)^{\gamma} \backslash \bar{G}(F)$. Thus our formula for the trace on the cuspidal part is

$$
\begin{aligned}
\operatorname{Tr}\left(P_{\text {cusp }} R(\varphi) P_{\text {cusp }} A\right)= & \sum_{\substack{\text { oo } \gamma \\
\gamma \text { elliptic }}} \int_{\bar{G}(F)^{\gamma} \backslash \bar{G}\left(\mathbb{A}_{F}\right)} \varphi\left(x^{-1} \gamma x\right) \alpha(\operatorname{det} x) d x \\
& +(\text { other terms }) .
\end{aligned}
$$

Define

$$
F_{\varphi}(\gamma)=\int_{\bar{G}\left(\mathbb{A}_{F}\right)^{\gamma} \backslash \bar{G}\left(\mathbb{A}_{F}\right)} \varphi\left(x^{-1} \gamma x\right) \alpha(\operatorname{det} x) d x
$$

This function makes sense as a function on the elliptic subset of $G(F)$ that satisfies

$$
F_{\varphi}\left(g \gamma g^{-1}\right)=\alpha(\operatorname{det} g) F_{\varphi}(\gamma) .
$$

We shall relate the elliptic elements $\gamma \in G(F)$ for which $F_{\varphi}(\gamma) \neq 0$ to an embedded copy of $R_{E / F}\left(E^{\times}\right)$in $G$. If $\gamma \in G(F)$ is elliptic, its eigenvalues are not in $F^{\times}$but lie in some quadratic extension $K=F(\sqrt{m})$. Left multiplication by members of $K$ on the $F$ basis $\{1, \sqrt{m}\}$ exhibits $K$ as the matrices $\left(\begin{array}{cc}a & m b \\ b & a\end{array}\right)$ with coefficients in $F$. Then $R_{K / F}\left(K^{\times}\right)$is exhibited as an explicit algebraic subgroup $H_{K}$ of $G$, and $\gamma$ is conjugate to a member of $H_{K}$.

Let us observe that if there exists $y$ in the centralizer $G\left(\mathbb{A}_{F}\right)^{\gamma}$ with $\alpha(\operatorname{det} y) \neq 1$, then $F_{\varphi}(\gamma)=0$. This is immediate from (2.4).

For $\gamma$ elliptic, the centralizer of $\gamma$ in $G$ is isomorphic to $H_{K}$ for some quadratic extension $K / F$. Then $\operatorname{det} G\left(\mathbb{A}_{F}\right)^{\gamma}=N_{K / F}\left(K^{\times} \backslash \mathbb{A}_{K}^{\times}\right)$. For $\alpha$ to be trivial on this, we must have $N_{K / F}\left(K^{\times} \backslash \mathbb{A}_{K}^{\times}\right) \subset \operatorname{ker} \alpha$. But class field theory says that the norm group determines the quadratic extension, and therefore $K \cong E$. The group $H_{E}$ is just our given algebraic group $H$ up to canonical isomorphism, and $H(F)=E^{\times}$. The only members of $E^{\times}$that have eigenvalues in $F$ are the scalar matrices, i.e., the members of $Z(F) \cong F^{\times}$.

This proves the desired relationship: What we conclude from the above is that an elliptic $\gamma \in G(F)$ has $F_{\varphi}(\gamma)=0$ unless the member $\gamma$ is conjugate to a member of $E^{\times}$and is not scalar. The $\mathfrak{o}_{\gamma}$ 's in question are thus parametrized by the nontrivial cosets of the quotient $F^{\times} \backslash E^{\times}$. Two elements $e$ and $e^{\sigma}$ parametrize the same $\mathfrak{o}_{\gamma}$, but there is no other redundance. Combining this conclusion with (2.3), we obtain

$$
\operatorname{Tr}\left(P_{\text {cusp }} R(\varphi) P_{\text {cusp }} A\right)=\frac{1}{2} \operatorname{vol}\left(\left(\mathbb{A}_{F}^{\times} E^{\times}\right) \backslash \mathbb{A}_{E}^{\times}\right) \sum_{\gamma \in\left(F^{\times} \backslash E^{\times}\right)-F^{\times}} F_{\varphi}(\gamma)+\text { (other terms). }
$$

The first term on the right side of (2.6) looks something like one side of the Poission summation formula for the group $\left(\mathbb{A}_{F}^{\times} E^{\times}\right) \backslash \mathbb{A}_{E}^{\times}$and its cocompact discrete subgroup $F^{\times} \backslash E^{\times}$, except that one term is missing. The Poisson summation formula 
is what the trace formula comes down to in the abelian case, and thus we have an indication of where we are headed.

Let us now refine matters by taking the "other terms" into account. Qualitatively the trace formula for $G$, when modified to handle $R(\varphi) A$, says that

$$
(\text { spectral terms })=(\text { geometric terms })
$$

and more specifically that

$$
\begin{gathered}
\left(\begin{array}{c}
\text { cuspidal } \\
\text { terms }
\end{array}\right)+\left(\begin{array}{c}
\text { continuous } \\
\text { terms }
\end{array}\right)+\left(\begin{array}{c}
\text { one-dimensional } \\
\text { terms }
\end{array}\right)+\left(\begin{array}{c}
\text { residual } \\
\text { terms }
\end{array}\right) \\
=\left(\begin{array}{c}
\text { central } \\
\text { terms }
\end{array}\right)+\left(\begin{array}{c}
\text { elliptic } \\
\text { terms }
\end{array}\right)+\left(\begin{array}{c}
\text { hyperbolic } \\
\text { terms }
\end{array}\right)+\left(\begin{array}{c}
\text { unipotent } \\
\text { terms }
\end{array}\right) .
\end{gathered}
$$

Here (2.2) gives

$$
\left(\begin{array}{c}
\text { cuspidal } \\
\text { terms }
\end{array}\right)=\operatorname{Tr}\left(P_{\text {cusp }} R(\varphi) P_{\text {cusp }} A\right)=\sum_{\substack{\Pi \cong \Pi \otimes \alpha, \Pi \text { cuspidal }}} \operatorname{Tr}\left(\Pi(\varphi) A_{\Pi}\right),
$$

and we have just seen that

$$
\left(\begin{array}{c}
\text { elliptic } \\
\text { terms }
\end{array}\right)=\frac{1}{2} \operatorname{vol}\left(\left(\mathbb{A}_{F}^{\times} E^{\times}\right) \backslash \mathbb{A}_{E}^{\times}\right) \sum_{\gamma \in\left(F^{\times} \backslash E^{\times}\right)-F^{\times}} F_{\varphi}(\gamma) .
$$

An argument like the one above that proves some vanishing for $F_{\varphi}(\gamma)$ shows that the central terms are 0 , and similar considerations show that the hyperbolic terms are 0 . A one-dimensional representation is never equal to its own nontrivial twist, and hence the one-dimensional terms are 0 . Further argument shows that the continuous terms are 0 . Thus our refined version of (2.6) is

$$
\begin{aligned}
& \sum_{\substack{\Pi \cong \Pi \otimes \alpha, \Pi \text { cuspidal }}} \operatorname{Tr}\left(\Pi(\varphi) A_{\Pi}\right)+\left(\begin{array}{c}
\text { residual } \\
\text { terms }
\end{array}\right) \\
& =\frac{1}{2} \operatorname{vol}\left(\left(\mathbb{A}_{F}^{\times} E^{\times}\right) \backslash \mathbb{A}_{E}^{\times}\right) \sum_{\gamma \in\left(F^{\times} \backslash E^{\times}\right)-F^{\times}} F_{\varphi}(\gamma)+\left(\begin{array}{c}
\text { unipotent } \\
\text { terms }
\end{array}\right) .
\end{aligned}
$$

We would like to be able to apply the Poisson summation formula to the first term on the right side of (2.7). Thus we extend $F_{\varphi}$ to a function on the set of regular elements in $\mathbb{A}_{E}^{\times}$(i.e., those elements having no component central), using formula (2.4). It turns out (see [Lab-L]) that $F_{\varphi}$ extends to a smooth function on $\mathbb{A}_{E}^{\times}$. However, its value on a central element is not given by (2.4) but rather by the unipotent terms of (2.7). Application of the Poisson summation formula therefore gives

$$
\sum_{\substack{\Pi \cong \Pi \otimes \alpha, \Pi \text { cuspidal }}} \operatorname{Tr}\left(\Pi(\varphi) A_{\Pi}\right)+\left(\begin{array}{c}
\text { residual } \\
\text { terms }
\end{array}\right)=\frac{1}{2} \operatorname{vol}\left(\left(\mathbb{A}_{F}^{\times} E^{\times}\right) \backslash \mathbb{A}_{E}^{\times}\right) \sum_{\chi \in\left(\left(\mathbb{A}_{F}^{\times} E^{\times} \backslash \mathbb{A}_{E}^{\times}\right)^{\wedge}\right.} \widehat{F_{\varphi}}(\chi) .
$$

Let $\left\{\chi, \chi^{\sigma}\right\}$ be given with $\chi \neq \chi^{\sigma}$. The same style of argument as at the end of $\S 1$ allows us to find a term on the left side corresponding to some $\Pi$ such that this term equals the sum of the terms for $\left\{\chi, \chi^{\sigma}\right\}$ on the right side. Then $\Pi$ is automorphically induced from some Grossencharacter depending on $\chi$. Actually 
the Grossencharacter is of the form $\theta=\chi \rho$ for a certain $\rho$ independent of $\chi$. We omit the details. (Cf. [Lap-R] for a similar argument in a slightly different context.)

The proof yields a bonus. Comparison of what terms are left after the above matching shows that the cuspidal $\Pi$ 's with $\Pi \cong \Pi \otimes \alpha$ are exactly the cuspidal $\Pi$ 's that are automorphically induced. This result is due to [Lab-L].

\section{Base Change}

Base change is described in $[R o 4, \S 16]$. For an extension $E / F$, the point of departure is the question whether restriction of Galois representations from $G_{F}$ to $G_{E}$ has a counterpart for automorphic representations. It is explained in [Kn1, §10] how this situation is an instance of global functoriality. Namely let

$$
H=G L_{n} / F \quad \text { and } \quad G=R_{E / F}\left(G L_{n} / E\right) .
$$

Here $G(F)=G L_{n}(E)$ and $G\left(\mathbb{A}_{F}\right)=G L_{n}\left(\mathbb{A}_{E}\right)$, and we have

$$
\begin{aligned}
{ }^{L} H & =G L_{2}(\mathbb{C}) \times \operatorname{Gal}(\bar{F} / F), \\
{ }^{L} G & =\left(G L_{n}(\mathbb{C}) \times \cdots \times G L_{n}(\mathbb{C})\right) \rtimes \operatorname{Gal}(\bar{F} / F),
\end{aligned}
$$

with the Galois group operating by permutations on the factors in the second case. The map ${ }^{L} H \rightarrow{ }^{L} G$ is given by the diagonal map on the identity component and by the identity map on the Galois group.

Making the above notions precise in a direct way requires the Local Langlands Conjecture. But as in $[$ Ro4, $\S 16]$ we can rephrase the question in such a way that it is not necessary to assume the Local Langlands Conjecture: Write the given automorphic representation $\pi$ of $H\left(\mathbb{A}_{F}\right)=G L_{n}\left(\mathbb{A}_{F}\right)$ as $\pi=\bigotimes_{v} \pi_{v}$ with $\pi_{v}$ equal to an unramified principal series for almost every place $v$ of $F$. An irreducible admissible representation $\Pi=\bigotimes_{w} \Pi_{w}$ of $G(\mathbb{A})_{F}=G L_{n}\left(\mathbb{A}_{E}\right)$ is said to be a base change lift of $\pi$ if it is automorphic and if, for almost all $v$ and all $w$ dividing $v, \pi_{v}$ is isomorphic to the Langlands subquotient of an unramified principal series induced from the character $\chi$ and $\Pi_{w}$ is isomorphic to the Langlands subquotient of an unramified principal series induced from the character $\chi^{\left[E_{w}: F_{v}\right]}$. These conditions determine $\Pi$ uniquely by strong multiplicity one. When $\Pi$ and $\pi$ are related in this way, we write $\Pi=B C_{E / F}(\pi)$.

Theorem 3. Let $E / F$ be a cyclic extension of prime degree $l$. If $\pi$ is a cuspidal representation of $G L_{n}\left(\mathbb{A}_{F}\right)$, then $\Pi=B C_{E / F}(\pi)$ exists as an automorphic representation of $G L_{n}\left(\mathbb{A}_{E}\right)$. Moreover, $\Pi$ is cuspidal unless $l$ divides $n$ and $\pi \otimes \alpha \cong \pi$ for some nontrivial character $\alpha$ of $F^{\times} \backslash \mathbb{A}_{F}^{\times}$that is trivial on $N_{E / F}\left(E^{\times} \backslash \mathbb{A}_{E}^{\times}\right)$.

In this generality Theorem 3 is due to Arthur and Clozel [Ar-Cl]. See [Ro4] for a more complete statement. We confine our comments to the special case that $n=2$, which was the case handled originally by Saito, Shintani, and Langlands [Lgl2]. For simplicity we shall assume $l \neq 2$. Thus write $H=G L_{2} / F$ and $G=R_{E / F}\left(G L_{2} / E\right)$, so that $H\left(\mathbb{A}_{F}\right)=G L_{2}\left(\mathbb{A}_{F}\right)$ and $G\left(\mathbb{A}_{F}\right)=G L_{2}\left(\mathbb{A}_{E}\right)$. The comparison of traces that leads to our special case of Theorem 3 involves the usual trace formula for $G L_{2}\left(\mathbb{A}_{F}\right)$ and a "twisted trace formula" for $G L_{2}\left(\mathbb{A}_{E}\right)$.

To describe this variant, we introduce the notion of twisted conjugacy of elements of $G L_{2}(E)$. By assumption, $\operatorname{Gal}(E / F)$ is cyclic of prime order $l$. Let $\sigma$ be a generator. Define $g$ and $g^{\prime}$ in $G L_{2}(E)$ to be $\sigma$ conjugate if there exists $x \in G L_{2}(E)$ 
such that $x^{-1} g \sigma(x)=g^{\prime}$. The associated norm map $N: G L_{2}(E) \rightarrow G L_{2}(E)$ is defined by $N(g)=g \sigma(g) \cdots \sigma^{l-1}(g)$. It is easy to check that

(1) $N\left(x^{-1} g \sigma(x)\right)=x^{-1} N(g) x$, and hence the $G L_{2}(E)$ conjugacy class of $N(g)$ equals $N$ of the $\sigma$ conjugacy class of $g$,

(2) $\sigma(N(g))=g^{-1} N(g) g$,

(3) the $G L_{2}(E)$ conjugacy class of $N(g)$ always meets $G L_{2}(F)$ in a unique $G L_{2}(F)$ conjugacy class.

Fact (2) shows that the trace and determinant of $N(g)$ belong to $F$, and (3) follows from this. Define

$$
\eta:\left\{\sigma \text { conjugacy classes in } G L_{2}(E)\right\} \rightarrow\left\{\text { conjugacy classes in } G L_{2}(F)\right\}
$$

by $\delta \mapsto \gamma$ if $\gamma \in G L_{2}(F)$ is $G L_{2}(E)$ conjugate to $N(\delta)$. This map can be shown to be injective, and we write $\gamma \in \eta(\delta)$. If $\delta$ is in $G L_{2}(E)$, the $\sigma$ centralizer of $\delta$ is an algebraic subgroup $G_{\sigma}^{\delta}$ of $G$ whose $F$ points are

$$
G_{\sigma}^{\delta}(F)=\left\{x \in G L_{2}(E) \mid x^{-1} \delta \sigma(x)=\delta\right\} .
$$

Let $Z$ be the group of 2 -by-2 scalar matrices, and put

$$
Z_{E}\left(\mathbb{A}_{F}\right)=Z(F) N_{E / F}\left(Z\left(\mathbb{A}_{E}\right)\right) .
$$

Fix a character $\omega$ of $Z_{E}\left(\mathbb{A}_{F}\right)$ trivial on $Z(F)$ and let $\omega_{E}$ be the character $z \mapsto \omega\left(N_{E / F}(z)\right)$ of $Z\left(\mathbb{A}_{E}\right)$. The relevant $L^{2}$ spaces will be $L_{E}^{2}$, built from $G$, and $L_{F}^{2}$, built from $H$ :

$$
\begin{aligned}
& L_{E}^{2}=\left\{f(z g)=\omega_{E}(z) f(g) \text { for all }\left.z \in Z\left(\mathbb{A}_{E}\right)\left|\int_{Z\left(\mathbb{A}_{E}\right) G L_{2}(E) \backslash G L_{2}\left(\mathbb{A}_{E}\right)}\right| f\right|^{2}<\infty\right\}, \\
& L_{F}^{2}=\left\{f(z g)=\omega(z) f(g) \text { for all }\left.z \in Z_{E}\left(\mathbb{A}_{F}\right)\left|\int_{Z_{E}\left(\mathbb{A}_{F}\right) G L_{2}(F) \backslash G L_{2}\left(\mathbb{A}_{F}\right)}\right| f\right|^{2}<\infty\right\} .
\end{aligned}
$$

Let $R_{E}$ and $R_{F}$ be the right regular representations of $G L_{2}\left(\mathbb{A}_{E}\right)$ and $G L_{2}\left(\mathbb{A}_{F}\right)$, respectively, on these $L^{2}$ spaces, and define an operator $A_{\sigma}$ on the first such space by $\left(A_{\sigma} F\right)(g)=F\left(\sigma^{-1} g\right)$. We are interested in the trace of $P_{\text {cusp }} R_{E}(\varphi) P_{\text {cusp }} A_{\sigma}$. is

As in $\S 2$ we write down the kernel of the unprojected operator $R_{E}(\varphi) A_{\sigma}$, which

$$
K(x, y)=\sum_{\delta \in \overline{G L}_{2}(E)} \varphi\left(x^{-1} \delta \sigma(y)\right)
$$

if we denote $Z \backslash G L_{2}$ by $\overline{G L}_{2}$. This operator is not of trace class, but we write down its (divergent) integral over the diagonal anyway, retaining the elliptic terms. The result is

$$
\begin{aligned}
& \operatorname{Tr}\left(P_{\text {cusp }} R_{E}(\varphi) P_{\text {cusp }} A_{\sigma}\right) \\
& \quad=\sum_{\substack{\{\delta\}, \sigma \text { conjugacy class, } \\
N \delta \text { elliptic }}} m_{\delta}^{\sigma} \int_{G_{\sigma}^{\delta}\left(\mathbb{A}_{F}\right) \backslash G L_{2}\left(\mathbb{A}_{E}\right)} \varphi\left(g^{-1} \delta \sigma(g)\right) d g+(\text { other terms }) .
\end{aligned}
$$


We want to compare (3.1) with

$$
\begin{aligned}
\operatorname{Tr}\left(P_{\text {cusp }} R_{F}(f) P_{\text {cusp }}\right) & \\
= & \sum_{\substack{\{\gamma\}, \gamma \text { elliptic }}} m_{\gamma} \int_{G L_{2}^{\gamma}\left(\mathbb{A}_{F}\right) \backslash G L_{2}\left(\mathbb{A}_{F}\right)} f\left(g^{-1} \gamma g\right) d g+(\text { still other terms). }
\end{aligned}
$$

The functions $\varphi$ and $f$ that we shall consider will be on $G\left(\mathbb{A}_{E}\right)$ and $G\left(\mathbb{A}_{F}\right)$, respectively, transforming under the central subgroups $Z\left(\mathbb{A}_{E}\right)$ and $Z_{E}\left(\mathbb{A}_{F}\right)$ oppositely to the members of the $L^{2}$ spaces, and being smooth of compact support modulo the central subgroups. We shall assume that $\varphi$ and $f$ are given by products of functions $\varphi_{v}$ and $f_{v}$ corresponding to the places of $F$ such that

(i) $\varphi_{v}$ is a function on $G L_{2}\left(E_{v}\right)$, smooth and compactly supported modulo $Z\left(E_{v}\right)$

(ii) $\varphi(z g)=\omega_{E}^{-1}(z) \varphi_{v}(g)$ for $z \in Z\left(E_{v}\right)$

and

(i') $f_{v}$ is a function on $G L_{2}\left(F_{v}\right)$, smooth and compactly supported modulo $Z\left(F_{v}\right)$

(ii') $f(z g)=\omega^{-1}(z) f_{v}(g)$ for $z \in N_{E_{v} / F_{v}}\left(Z\left(E_{v}\right)\right)$.

The idea is to show that

$$
l \operatorname{Tr}\left(P_{\text {cusp }} R_{E}(\varphi) P_{\text {cusp }} A_{\sigma}\right)=\operatorname{Tr}\left(P_{\text {cusp }} R_{F}(f) P_{\text {cusp }}\right)
$$

whenever $\varphi$ and $f$ are suitably compatible. In the first place one shows that $l m_{\delta}^{\sigma}=$ $m_{\gamma}$ if $\gamma \in \eta(\delta)$; this is a Tamagawa-number problem that we shall not discuss.

In the second place one is to show that every $\varphi$ gives a compatible $f$. The meaning of compatibility is that we can associate $\varphi_{v} \rightarrow f_{v}$ for all $v$ in such a way that, for all regular semisimple $\gamma \in G L_{2}(F)$,

$$
\int_{G L_{2}^{\gamma}\left(\mathbb{A}_{F}\right) \backslash G L_{2}\left(\mathbb{A}_{F}\right)} f\left(g^{-1} \gamma g\right) d g
$$

is 0 if $\gamma$ is not a norm from $G L_{2}(E)$ and is

$$
\int_{G_{\sigma}^{\delta}\left(\mathbb{A}_{F}\right) \backslash G L_{2}\left(\mathbb{A}_{E}\right)} \varphi\left(g^{-1} \delta \sigma(g)\right) d g
$$

if $\gamma \in \eta(\delta)$. Early work with this notion is due to Saito and Shintani.

Showing that every $\varphi$ gives an $f$ is a manageable problem in local analysis. But even when we solve this problem, we have not solved the base change problem. In applying the trace formulas, we have to choose $\varphi_{v}$ and $f_{v}$ at almost every place to be the identity in the appropriate bi-K-invariant Hecke algebras ( $K$ being a standard maximal compact subgroup). Thus we have to know for almost every place that if $\varphi_{v}$ is the normalized characteristic function of the maximal compact subgroup of $G L_{2}\left(E_{v}\right)$, then $f_{v}$ can be taken to be the normalized characteristic function of the maximal compact subgroup of $G L_{2}\left(F_{v}\right)$.

This is a special case of a result that is mostly conjectural and is known as the fundamental lemma. Its importance is emphasized in the lectures [Lgl4] of Langlands. See Kottwitz [Ko1] for the fundamental lemma in the case of base change for $G L_{2}$. For generalizations see [Ko2], [Ar-Cl], [Lab2], [Wa1], and [Ha]. 
The result is that the main terms on the right sides of (3.1) and (3.2) are equal (apart from the factor of $l$ ). The hard part is to show that the contributions from the other terms cancel, so that (3.3) results. This is done in [Lgl2, §11]. The proof that (3.3) implies our case of Theorem 3 is summarized on [Lgl, p. 20], and the details are carried out in $[\operatorname{Lgl} 2, \S 11]$.

\section{Applications Involving a Single Group}

We discuss briefly in this section three applications of the trace formula of a single group.

4.1. Eichler-Selberg trace formula. This theorem in its classical form is derived in [Ei2], and an exposition appears in [Mi]. We use the standard terminology of [Shi2]. Let $N>1$ be an integer, and let $\Gamma_{0}(N)$ be the subgroup of $S L_{2}(\mathbb{Z})$ of matrices whose lower left entry is divisible by $N$. Let $S_{k}(N)$ be the space of (analytic) cusp forms of weight $k$ relative to $\Gamma_{0}(N)$. This space is known to be finitedimensional. If $p$ is a prime not dividing $N$, then the Hecke operator $T_{p}$ is defined on, among other things, the space $S_{k}(N)$. The Eichler-Selberg trace formula computes the trace of this operator. If $p$ is put equal to 1 in the formula, the operator $T_{p}$ becomes the identity, and the formula gives an expression for the dimension of $S_{k}(N)$. (This dimension formula may also be derived from the Riemann-Roch Theorem.)

The Eichler-Selberg trace formula may also be derived from the (adelic) trace formula for $G L_{2}$, with the character $\omega$ of the center taken to be 1 . This kind of proof has been carried out in [Du-La], although it is not immediately obvious how to correlate the terms of the $G L_{2}$ trace formula in [Du-La] with the terms in Theorem 7.14 of [Kn2]. Let us take $N=1$ for simplicity. The space $S_{k}(1)$ is spanned by simultaneous eigenfunctions of all the Hecke operators, and each such eigenfunction gives rise to a cuspidal automorphic representation $\pi=\pi_{\infty} \otimes \bigotimes_{p} \pi_{p}$ whose component $\pi_{\infty}$ at the infinite place is a discrete series with extreme weight $\pm k$. Let $\varphi=\varphi_{\infty} \times \prod_{p} \varphi_{p}$ be defined as follows. For finite $p, \varphi_{p}$ modulo center is just the characteristic function of the usual maximal compact subgroup of $G L_{2}\left(\mathbb{Q}_{p}\right)$. For $\infty, \varphi_{\infty}$ is the matrix coefficient $\left(\pi_{\infty}(\cdot) v, v\right)$, where $v$ has extreme weight. The idea is that the trace formula for $G L_{2}$ is to be applied to this $\varphi$.

The difficulty is that this $\varphi$ does not have compact support modulo center. At this point one can apply the main result of [Lab3], which creates a compactly supported function behaving like the function $\varphi$ above and having constant term 0 . Then the $G L_{2}$ trace formula is applicable, and the formula for the trace of the Hecke operator follows.

For a generalization of this theorem, see $[\operatorname{Ar} 1]$.

4.2. An application to quaternion algebras. Let $F$ be a number field, and let $E / F$ be a quadratic extension. By class field theory there exists a nontrivial character $\alpha$ of $F^{\times} \backslash \mathbb{A}_{F}^{\times}$such that $\operatorname{ker} \alpha=N_{E / F}\left(E^{\times} \backslash \mathbb{A}_{E}^{\times}\right)$. Let $D$ be a quaternion (division) algebra over $F$, let $G^{\prime}$ be the multiplicative group, and suppose that $E$ does not embed in $D(F)$. Then the theorem is that $\pi^{\prime} ¥ \pi^{\prime} \otimes \alpha$ for every automorphic representation occurring in $L^{2}\left(G^{\prime}, \omega\right)$.

The argument runs parallel to that for Theorem 2 . Let $R$ be the right regular representation of $G^{\prime}\left(\mathbb{A}_{F}\right)$ on $L^{2}\left(G^{\prime}, \omega\right)$. Define an operator $A: L^{2}\left(G^{\prime}, \omega\right) \rightarrow L^{2}\left(G^{\prime}, \omega\right)$ 
by $A f(x)=f(x) \alpha(\operatorname{det} x)$. Then $A$ satisfies the intertwining relation

$$
A(R \otimes(\alpha \circ \operatorname{det}))(x) f=R(x) A f \quad \text { for } f \in L^{2}\left(G^{\prime}, \omega\right) .
$$

By the Corollary to Theorem 1b, the automorphic representations in $L^{2}\left(G^{\prime}, \omega\right)$ have multiplicity one. Arguing as in $\S 2$, we obtain

$$
\operatorname{Tr}(R(\varphi) A)=\sum_{\substack{\pi^{\prime} \cong \pi^{\prime} \otimes \alpha, \pi^{\prime} \text { automorphic }}} \operatorname{Tr}\left(\pi^{\prime}(\varphi) A_{\pi^{\prime}}\right),
$$

where $A_{\pi^{\prime}}$ is the restriction of $A$ to the space of $\pi^{\prime}$.

The operator $R(\varphi) A$ is an integral operator with kernel

$$
\sum_{\gamma \in \bar{G}^{\prime}(F)} \varphi\left(x^{-1} \gamma y\right) \alpha(\operatorname{det} y) .
$$

Since $Z^{\prime}\left(\mathbb{A}_{F}\right) G^{\prime}(F) \backslash G^{\prime}\left(\mathbb{A}_{F}\right)$ is compact, $R(\varphi) A$ is of trace class, and its trace is

$$
\operatorname{Tr}(R(\varphi) A)=\sum_{\mathfrak{o}_{\gamma}} \int_{\bar{G}^{\prime}(F)^{\gamma} \backslash \bar{G}^{\prime}\left(\mathbb{A}_{F}\right)} \varphi\left(x^{-1} \gamma x\right) \alpha(\operatorname{det} x) d x,
$$

where $\mathfrak{o}_{\gamma}$ consists of all $\delta^{-1} \gamma \delta$ with $\delta$ varying through $\bar{G}^{\prime}(F)^{\gamma} \backslash \bar{G}^{\prime}(F)$.

Define

$$
F_{\varphi}(\gamma)=\int_{\bar{G}^{\prime}\left(\mathbb{A}_{F}\right)^{\gamma} \backslash \bar{G}^{\prime}\left(\mathbb{A}_{F}\right)} \varphi\left(x^{-1} \gamma x\right) \alpha(\operatorname{det} x) d x
$$

for $\gamma \in \bar{G}^{\prime}(F)$. If $\gamma \in \bar{G}^{\prime}(F)$ is 1 , this is 0 . Otherwise $\gamma \in \bar{G}^{\prime}(F)$ lies in a unique quadratic extension $K$ within $D(F)$, and the centralizer consists of the nonzero elements of this quadratic extension. Since $K$ cannot be $E$ by hypothesis, an argument in $\S 2$ shows that $F_{\varphi}(\gamma)=0$ in this case. Consequently $\operatorname{Tr}(R(\varphi) A)=0$. Then

$$
\sum_{\substack{\pi^{\prime} \cong \pi^{\prime} \otimes \alpha, \pi^{\prime} \text { automorphic }}} \operatorname{Tr}\left(\pi^{\prime}(\varphi) A_{\pi^{\prime}}\right)=0 .
$$

A generalized independence argument allows us to conclude that the sum is empty, and the theorem follows.

4.3. A consequence of the Eichler-Shimura congruence relation. This theorem in its classical form is derived in [Ei1] and [Shi1] and explained in [Sw-Bi]. It yields a formula for the $L$ function of $X_{0}(N)$, the modular curve attached to the group $\Gamma_{0}(N)$ defined in $\S 4.1$, as a product of $L$ functions of cuspidal representations of weight 2. This product formula, place by place, is a consequence of the trace formula for $G L_{2}$. See [Ih]. For a generalization to a group other than $G L_{2}$, see [Lgl-Ra]. For an exposition see [Bl-Ro].

\section{References}

[Ar1] Arthur, J., The $L^{2}$-Lefschetz numbers of Hecke operators, Invent. Math. 97 (1981), $257-290$.

[Ar2] Arthur, J., Stability and endoscopy: Informal motivation, these Proceedings, pp. 443-442.

[Ar-Cl] Arthur, J., and L. Clozel, Simple Algebras, Base Change, and the Advanced Theory of the Trace Formula, Princeton University Press, Princeton, 1989. 
[Bl-Ro] Blasius, D., and J. Rogawski, Zeta functions of Shimura varieties, Motives (U. Jannsen, S. Kleiman, J.-P. Serre, eds.), Proc. Symp. Pure Math., vol. 55, Part II, American Mathematical Society, Providence, 1994, pp. 525-571.

[DKV] Deligne, P., D. Kazhdan, and M.-F. Vigneras, Représentations des Algèbres Centrales Simples p-adiques, Représentations des Groupes Réductifs sur un Corps Local (J.-N. Bernstein, P. Deligne, D. Kazhdan, and M.-F. Vigneras, eds.), Travaux en Cours, Hermann, Paris, 1984, pp. 33-117.

[Du-La] Duflo, M., and J.-P. Labesse, Sur la formule des traces de Selberg, Annales Sci. École Norm. Sup. 4 (1971), 193-284.

[Ei1] Eichler, M., Quaternäre quadratische Formen und die Riemannsche Vermutung fr̈ die Kongruenzzetafunktionen, Arch. der Math. 5 (1954), 355-366.

[Ei2] Eichler, M., Eine Verallgemeinerung der Abelschen Integrale, Math. Zeitschr. 67 (1957), 267-298.

[Gb1] Gelbart, S. S., Automorphic Forms on Adele Groups, Princeton University Press, Princeton, NJ, 1975.

[Gb-Ja] Gelbart, S., and H. Jacquet, Forms of GL(2) from the analytic point of view, Automorphic Forms, Representations, and L-Functions (A. Borel and W. Casselman, eds.), Proc. Symp. Pure Math., vol. 33, Part I, American Mathematical Society, Providence, 1979, pp. 213-251.

[Ha] Hales, T. C., On the fundamental lemma for standard endoscopy: reduction to unit elements, Canad. J. Math. 47 (1995), 974-994.

[Ih] Ihara, Y., Hecke polynomials as congruence $\zeta$ functions in elliptic modular case, Annals of Math. 85 (1967), 267-295.

[Ja] Jacquet, H., Automorphic spectrum of symmetric spaces, these Proceedings, pp. $443-455$.

[Ja-Lgl] Jacquet, H., and R. P. Langlands, Automorphic Forms on GL(2), Lecture Notes in Mathematics, vol. 114, Springer-Verlag, Berlin, 1970.

[Kn1] Knapp, A. W., Introduction to the Langlands program, these ProceEdings, pp. 245-302.

[Kn2] Knapp, A. W., Theoretical aspects of the trace formula for $G L(2)$, these ProceEdings, pp. 355-405.

[Ko1] Kottwitz, R., Orbital integrals and base change, Automorphic Forms, Representations, and L-Functions, Proc. Symp. Pure Math., vol. 33, Part II, American Mathematical Society, Providence, 1979, pp. 111-113.

[Ko2] Kottwitz, R. E., Base change for unit elements of Hecke algebras, Compositio Math. 60 (1986), 237-250.

[Lab1] Labesse, J.-P., $L$-indistinguishable representations and trace formula for $S L(2)$, Lie Groups and Their Representations: Summer School of the Bolyai János Mathematical Society (Budapest 1971) (I. M. Gelfand, ed.), John Wiley \& Sons, New York, 1975, pp. 331-338.

[Lab2] Labesse, J.-P., Fonctions élémentaires et lemme fondamental pour le changement de base stable, Duke Math. J. 61 (1990), 519-530.

[Lab3] Labesse, J.-P., Pseudo-coefficients trés cuspidaux et $K$ théorie, Math. Annalen 291 (1991), 607-616.

[Lab-L] Labesse, J.-P., and R. P. Langlands, L-indistinguishability for SL(2), Canad. J. Math. 31 (1979), 726-785.

[Lap-R] Lapid, E., and J. Rogawski, On twists of cuspidal representations of $G L(2)$, Forum Math. (to appear).

[Lg11] Langlands, R. P., On the Functional Equations Satisfied by Eisenstein Series, Lecture Notes in Mathematics, vol. 544, Springer-Verlag, Berlin, 1976.

[Lgl2] Langlands, R. P., Base Change for GL(2), Princeton University Press, Princeton, 1980.

[Lgl3] Langlands, R. P., Les Débuts d'une Formule des Traces Stable, Publications Mathématiques, vol. 13, L'Université Paris VII, Paris, 1983.

[Lgl4] Langlands, R. P., Where stands functoriality today?, these Proceedings, pp. 457-471.

[Lgl-Ra] Langlands, R. P., and D. Ramakrishnan (eds.), The Zeta Functions of Picard Modular Surfaces, Les Publications CRM, Montréal, 1992.

[Mi] Miyake, T., Modular Forms, Springer-Verlag, Berlin, 1989. 
[Mo-Wa] Moeglin, C., and J.-L. Waldspurger, Décomposition Spectrale et Séries d'Eisenstein, Birkhäuser Verlag, Basel, 1994.

[Ra] Ramakrishnan, D., Pure motives and automorphic forms, Motives (U. Jannsen, S. Kleiman, J.-P. Serre, eds.), Proc. Symp. Pure Math., vol. 55, Part II, American Mathematical Society, Providence, 1994, pp. 411-446.

[Ro1] Rogawski, J. D., Representations of GL $(n)$ and division algebras over a $p$-adic field, Duke Math. J. 50 (1983), 161-196.

[Ro2] Rogawski, J. D., Automorphic Representations of Unitary Groups in Three Variables, Princeton University Press, Princeton, 1990.

[Ro3] Rogawski, J. D., Modular forms, the Ramanujan conjecture and the Jacquet-Langlands correspondence, Discrete Groups, Expanding Graphs and Invariant Measures, by A. Lubotzky, Birkhäuser, Basel, 1994, pp. 135-176.

[Ro4] Rogawski, J. D., Functoriality and the Artin conjecture, these Proceedings, pp. 331-353.

[She] Shelstad, D., Orbital integrals for $\mathrm{GL}_{2}(\mathbf{R})$, Automorphic Forms, Representations, and L-Functions, Proc. Symp. Pure Math., vol. 33, Part I, American Mathematical Society, Providence, 1979, pp. 107-110.

[Shi1] G. Shimura, Correspondances modulaires et les fonctions $\zeta$ de courbes algébriques, $J$. Math. Soc. Japan 10 (1958), 1-28.

[Shi2] G. Shimura, Introduction to the Arithmetic Theory of Automorphic Functions, Princeton University Press, Princeton, NJ, 1971.

[Sw-Bi] Swinnerton-Dyer, H. P. F., and B. J. Birch, Elliptic curves and modular functions, Modular Functions of One Variable IV, Lecture Notes in Mathematics, vol. 476, SpringerVerlag, Berlin, 1975, pp. 2-32.

[Vi] Vigneras, M.-F., Arithmétique des Algèbres de Quaternions, Lecture Notes in Math., vol. 800, Springer-Verlag, Berlin, 1980.

[Wa1] Waldspurger, J.-L., Sur les intégrales orbitales tordues pour les groupes linéaires: un lemme fondamental, Canad. J. Math. 43 (1991), 852-896.

[Wa2] Waldspurger, J.-L., Homogénéité de certains distributions sur les groupes $p$-adiques, Publ. Math. I.H.E.S. 81 (1995), 25-72.

Department of Mathematics, State University of New York, Stony Brook, New York 11794, U.S.A.

E-mail address: aknapp@ccmail.sunysb.edu

Department of Mathematics, The Hebrew University, Givat Ram, Jerusalem, Israel

E-mail address: jonr@math.huji.ac.il 